\title{
ANALISIS DE DOS MODELOS EVALUATIVOS REFERIDOS A LA QUIMICA DE COU Y SELECTIVIDAD (primeros resultados)
}

\author{
LOPEZ, N.; LLOPIS, R.; LLORENS, J.A.; SALINAS, B.: SOLER, J.
}

\section{SUMMARY}

This article is about the content and analysis of examinations and assessment in a specific field: the teaching of Chemistry.

We compare the results obtained by students in different internal test during and the end of an acadernic year with those obtained by the same students in an external test set by the entrance examination board at the University of Valencia.

\section{INTRODUCCION}

El tema de los exámenes, y de la evaluación en general, ya problemático de por sí en el campo de la ensehanza, cobra una especial emportancia cuando los resultados obtenidos en el propio examen sirven como parámetros para la promoción a un nivel de estudios académicos supcrior, tal como ocurre en la llamada "prueba de selectividad", utilizada como base para el posible acceso a la Universidad por parte del alumnaJo procedente del Curso de Orientación Universitaria (COU).

En cuanto a la prueba de selectividad, objeto de ruestro trabajo, digamos que sus planteamientos generales, más que responder a una necesidad estríctamente académica, responden a una necesidad para satisfacer la demanda inicial de admisión del alumno procedente de la enzehanza media.

De cualquier forma esta necesidad de selección adquiere un carácter eminentemente cualitativo cuando el ins. trumento utilizado para discriminar es un conjunto de pruebas que intentan ser representativas de algunos de los puntos fundamentales sobre los que el alumno de. beria haber trabajado, en la disciplina correspondienie. en sus anos de bachillerato y especialmente en C.O.U.

En el presente trabajo hemos intentado el análisis de la llamada "prueba de selectividad", referida al campo de la Química, desde dos aspectos fundamentales: contenidos exigidos y estructura formal de la prueba (1).

Nuestra opinión es que un exámen, una prueba de eva. luación, en su forma, en las conductas que exige, en el lenguaje que utiliza, etc. está dando una medida de la propia calidad de la enseñanza. Además en el mo. mento en que la prueba de selectividad se aleje, en sus planteamientos, de la estructura global planteada en el Bachillerato y C.O.U., puede darse un desfase total entre el criterio de evaluación utilizada en la enseñanza media, y el criterio utilizado para el posible acceso a la Universidad. Creemos que la representatividad de la prueba de selectividad ha de ser la base fundamental de la medida de la validez externa de la misma.

Para Klopfer, cuya taxonomia de objetivos referidos al campo de las ciencias, basada en la ya clástca taxonomia de Bloom y colegas, nos ha sido de gran utilidad, existen una serie de objetivos muy concretos que no pueden medirse simplemte a partir de una prueba, o de un tipo de pruebas. De hecho, Klopfer, a través de su taxonomia, nos está presentando todo un modelo de enseñanza de las ciencias qe conlleva una serie de actividades de laboratorios, de manipulación y observación que es preçiso evaluar (2). Quizás en una prueba escrisa esto es imposible, y en el caso de la selectividad este tipo de planteamientos escapan por completo a la estructura organizativa en la que se desenvuelve dicha prueba, sin embargo, la evaluación de las ciencias, y en concreto de la Quimica no deberia convertirse simplemente en una medición de conocimientos, sino también en un intento por evidenciar una actitud cientifica general por parte del alumno, donde quizás no importe tanto que se defina una ley como que se razone, como que se aplique en el momento oportuno, y que se verbatice a través de un lenguaje cientifico integrado y no repetitivo, en suma, que se ponga de manifiesto un aprendizaje eminentemente significativo (3).

Este tipo de aprendizaje, definido por Ausubel como "significativo", se encuentra en la base de nuestros planteamientos, tanto en el análisis que realizamos de los contenidos que deberian estar presentes en una prueba del tipo de la selectividad, como en los modelos de evaluación que presentamos y que están referidos al desarrollo global del curso de C.O.U. 


\section{PLANTEAMIENTO DEL PROBLEMA}

Los distintos modelos de evaluacion y los tipos de pruebas utilizados en la enseñanza están configurado en la actualidad el tipo de ensenanza desarrollada, acoplando en muchos casos la metodología y los objectivos a los procedimientos de las prueba de selectividad o de acceso a la Universidad, tanto en Quimica como en otras materias.

Durante los úlimos seis años hemos ido recopilando las pruebas de selectividad en Quimica de la Universidad de Valencia. Un anditsis inmediato de dichas pruebas pone de manifiesto la carencia de un modelo de evaluación válido y actualizado para la enseñanza de las ciencias.

El estudio comparativo de una serie de instituciones de Gran Bretana, Suiza, Estados Unidos y Francia, dedicadas a la preparación de exámenes a un nivel similar, nos decidieron a planificar esta investigación. En este trabajo presentamos los resultados de la fase inicial, aun siendo conscientes de sus limitaciones, por cuanto creemos son indicativos de la situacion real en la que se desenvuelve la selectividad en nuestro pais.

Intentamos también en el desarrollo de este trabajo, abordar muchos aspectos que la ensertanza de la Ciencia implica.

En el planteamiento de hipótesis que hacemos a continuación (referidas a la fase inicial de la investigacion) sólo pretendemos aproximarnos a lo que está ocurriendo actualmente en la prueba de selectividad. Enunciamos pues:

Hipótesis A. - Hemos aceptado como fiables los resultados obtenidos por cada alumno a lo largo del curso (prueba E, realizada en Mayo). En dichas pruebas $(A, B, E)$ se contempla casi todo el contenido y muchas de las capacidades que un alumno de Quimica de COU debe poseer. Además participaron en su redacción y revisión un amplio grupo de profesores de Fisica y Quimica de Bachiłlerato (aproximadamente quince). Por tanto la media de las pruebas A y B debe estar altamente correlacionada con los resultados de la prueba $E$, y no deben existir diferencias significativas entre $A$, B y $E$.

Hipotesis B. - Dadas tas características que la legislación vigente atribuye a la prueba de selectividad y a la especifica de Quimica, y el tiempo disponible (45 minutos), superpuesto a dos opciones a elegir, esta situación obliga a los coordinadores a preparar y proponer ejercicios cortos, con un número limitado de contenidos, características,... Por tanto creemos que los resultados de dichas pruebas de selectividad en Quimica, estarán poco correlacionados con la prueba $E$ y con la media de A y B. Por tanto, los resultados de la prueba de selectividad no son indices vallidos para evaluar el aprendizaje global de los alumnos en Quimica a lo largo de tres cursos (segundo y tercero de B.U.P. y
C.O.U.), siendo el azar, en muchos casos, el que pue. de determinar la nota de los alumnos en la prueba de selectividad.

\section{DISEÑO Y DESCRIPCION DE LA EXPE- RIENCIA DESARROLLADA}

\section{Proceso de elaboración de pruebas}

En la preparación de las pruebas concretas hemos tenido que partir de exámenes ajenos a la propia dinámica interna de los centros, intentado de esa forma, una cierta uniformidad a la hora de comparar los contenidos planteados en las pruebas.

A nivel internacional, los tipos de prueba que hemos encontrado en los diferentes paises, son muy distintos, en un extremo están los de la American Chemical Society (A.C.S.), que presentan exclusivamente cuestio. nes de elección múltiple, con una duración superior a una hora (unos ochenta minutos) y ochenta items de cuatro alternativas cada uno (4). En el orro extremo nos encontraríamos con los exámenes de acceso preparados por las distintas Universidades inglesas (5) en los que el numero y los tipos de cuestiones son muy variados. Veamos un ejemplo: el departamento de exámenes de la Universidad de Londres prepara el A-level Nuffield Chemistry, utilizado para el acceso a la ensehanza universitaria inglesa, está formado por:

- 50 cuestiones de elección mültiple, en una hora y cuarto

- una cuestión de los estudios especiales, en tres cuartos de hora

- tres cuestiones a elegit entre nueve, en una hora y tres cuartos

- ocho cuestiones, obligatorias, estructuradas, en dos horas

Lo que se está midiendo - evaluando- durante las cinco horas y tres cuartos en que se desarrollan las distintas sesiones de este examen es una serie de caracteríticas y contenidos, con lo cual pueden lograr un acercamiento a la situación real de conocimientos y capacidades de un alumno de Química.

Es evidente que con exámenes tan completos y con tal variedad de aspectos solicitados, se puede conocer la sicuación de los candidatos, lo que por otra parte, exige un tipo de enseñanza que haga posible tan diversificada capacitación.

Ya la comisión Lagarique (6), encargada de la reforma de la ensentanza de las ciencias en Francia indicaba: "Los aspectos docimilogicos se plantean con un especial cuidado dentro de las ciencias experimentales en concreto; es necesario consagrarles un estudio especials.

Haciéndose eco de esta necesidad surgio en Parts, en la Universidad de Paris VII, y dentro del 
"L.J.R.E.S.P.T." el Groupe Chapham (7), comisión encargada de analizar la evaluación de las Ciencias, y que propone cuatro tipos de cuestiones que deben aparecer en un control de la enseñanza de las Ciencias: (a) cuestiones sobre los métodos de razonamiento experimental, (b) problemas, (c) cuestiones de respuesta cor$\mathrm{ta}$, (d) lectura critica de un texto científico.

La distribución de tiempo sería: la mitad para la parte (b); un cuarto del tiempo para la parte (a); y otro cuarto del tiempo para las partes (c) o (d).

- el número de cuestiones es muy abundante, lo que permite evaluar los distintos contenidos, estando siempre presentes los más importantes y caracteristicos.

- Si se utilizan distintos tipos de cuestiones podremos evaluar una serie de capacidades que la ensentanza de la Quimica requiere. Por ejemplo, al plantear un problema estamos comprobando «las capacida. des de formalización, de cálculo, de sintesis» (8); pero este problema versará sobre un contenido concreto, por ejemplo: estequiometria, ácido-base, etc.. La bibliografia sobre distintos planteamientos de cuestiones o ilems en los examenes es abundante, en sintesis, el tipo de cuestión dependerá de lo que pretendemos medir. (9)

La A.C.S., aunque en sus examenes optan por pruebas de elección múlciple, propone como formas principales de cuestiones en el campo de la Quimica: (a) de verdadero-falso, (b) item por completar, (c) items de eleccion multiple, (d) pruebas de ensayo, (e) cuestiones estructuradaś, (n ejercicios prácicos, (g) resolución de problemas.

Desde nuestro punto de vista, lo más adecuado seria aplicar al alumno diferentes tipos de pruebas, y que los resultados de éstas sirvieran de confirmación a la nota o resultado final. Caso del examen de Londres antes cilado, puede estudiarse algún tipo de correlación existente entre cada prueba y el resultado final. Durante tres antos, un grupo de investigadores dirigido por el profesor John Mathew5, de la Universidad de Lancaster, ha estado estudiando todos los resultados de los alumnos que seguían la Quimica Nuffield a nive! avan. zado. Los tipos de pruebas que presentaron mayores indices de correlación con la calificación definitiva fueron las pruebas de elección múltiple y las pruebas con cuestiones cortas estructuradas, y las de menor corre. lación fueron las notas del trabajo práctico preparado por el profesor (10)

Nuestro propósito es establecer pruebas que en poco tiempo de ejecución abarquen un amplio espectro de contenidos y capacidades, cosa que permiten las pruebas de elección múltiple. El conjunto de cuestiones de elección múltiple, las de respuesta corta y los problemas, abarcan los aspectos más importantes del progra$\mathrm{ma}$, siguiendo las recomendaciones y resultados obtenidos por J. Mathews y colaboradores.
Nuestras pruebas se han elaborado a partir de pruebas externas de otros paises, (11), (12), (13): de manuales (14) y de items elaborados por nosolros. Todas las observaciones serán muy biẹn recibidas.

\section{Análisis de resultados}

Una vez delimitado el campo de hipótests, nos correspondia desarrollar un diseño sencilio con el fin de contrastar las hipótesis y problemas planteados.

La investigación que presentamos a continuación se concreta en un análisis de los resultados obtenidos por varios grupos de alumnos que siguen la Quimica en ei nivel C.O.U. y que una vez aprobado dicho curso, pasan a la prueba de selectividad, y en el soreo de la asignaturas optativas deben realizar la prueba de Quími. ca. A pesar de que las pruebas A, B y E se realizaron sobre una población de 300 alumnos, sólo 32 de ellos tuvieron que responder al examen $S$, examen de selectividad. Recordemos que A y $B$ son exámenes parciales, $E$ es el examen final que reure los contenidos de A y B, y $S$ es el examen de acceso (selectividad) pro. puesto por la Universidad de Valencia. La descripción de estos exámenes figura en el anexo 1.

Los estadisticos fundamentales que manejamos son:

Para conocer las relaciones entre estos tres grupos de calificaciones, se planeó por una parte el cálculo de la correlación de Bravais-Pearson o coeficiente "t". 10mando las muestras de dos en dos ("). En este procedimiento estadistico la coordinación de puntuaciones hay que formularla por referencia a los grados de 60 . rrespondencia (15).

Por otra parte, hemos utilizado un análisis de varianza "ANOVA», para determinar estadisticamente el grado de significación entre las medias de los tres grupos de puntuaciones (16).

Para comprobar otras hipotesis emitidas, que liguran en las perspectivas abiertas por esta investigación. será necesario mucho más tiempo y otros diseños pouteriores más ambiciosos.

Los resultados presentados a continuación son los on. tenidos por los alumnos antes citados.

TABLA I - Calificaciones oblenidas (*)

Alumno

\begin{tabular}{ccc}
\multicolumn{3}{c}{ Puniuaciones } \\
\hline $\begin{array}{c}\text { Media } \\
\text { Curso }\end{array}$ & $\begin{array}{c}\text { Exámen } \\
\text { final } \\
\text { "AB" }\end{array}$ & $\begin{array}{c}\text { Pruebs } \\
\text { Selectividad } \\
\text { "En" }\end{array}$ \\
\hline 5.2 & 4.6 & 55 \\
4.1 & 5.2 & 5
\end{tabular}

(*) Las expresiones que permiten conocer los coeficientes de corre lación son tales que "r» tiene un valor maximo de. 1 y el valor mínimo de -1 , correspondientes a la máxima y minuma correta. cion respectivamente. 


3
4
5
6
7
8
9
10
11
12
13
14
15
16
17
18
19
20
21
22
23
24
25
26
27
28
29
30
31
32
7

- Solo liguran las nolas de los alumnos aprobados en el conjunto de asignatutas de COU. Para pasar a la selectividad es necesario tener aprobado completo el COU.

TABLA 1I + Resultados estadisticos.

\begin{tabular}{|c|c|c|c|}
\hline \multirow[t]{2}{*}{ Estadistico } & \multicolumn{3}{|c|}{ PRUEBA } \\
\hline & $\begin{array}{l}\text { Curso } \\
\text { "AB" }\end{array}$ & $\begin{array}{c}\text { Exámen } \\
\text { Irinal } \\
\text { "E" }\end{array}$ & $\begin{array}{c}\text { Prueba } \\
\text { Selectividad } \\
\text { "Sw }\end{array}$ \\
\hline $\begin{array}{l}\text { MEDIA } \\
\text { DESV. TIPICA } \\
\text { RANGO }\end{array}$ & $\begin{array}{c}6.2 \\
1.47 \\
8.9 \cdot 3.4\end{array}$ & $\begin{array}{c}6.7 \\
1.66 \\
9.5 .3 .7\end{array}$ & $\begin{array}{r}3.9 \\
1.98 \\
1.5 \cdot 0\end{array}$ \\
\hline
\end{tabular}

TABLA III - Estadisticos entre grupos (•)

\begin{tabular}{|c|c|c|c|c|}
\hline PRUEBA & $\begin{array}{l}\text { Correlación } \\
\text { "rs Pearson }\end{array}$ & $\begin{array}{l}\text { Desviación } \\
\text { Máxima }\end{array}$ & $\begin{array}{c}\text { Desviación } \\
\text { Media }\end{array}$ & $\begin{array}{c}\text { Anova } \\
\text { "F" Fisher }\end{array}$ \\
\hline $\begin{array}{l}A B \cdot E \\
A B \cdot S\end{array}$ & $\begin{array}{l}.81 \\
.37\end{array}$ & $\begin{array}{l}2.2 \\
5.6\end{array}$ & $\begin{array}{r}.9 \\
2.4\end{array}$ & $\begin{array}{l}1.57 \\
26.5\end{array}$ \\
\hline 8 & .38 & 6.1 & 2.9 & 35.9 \\
\hline
\end{tabular}

(*) Aunque jos estadfsticos que se adjuntan corresponden a los 32 alumnos que reatizaron todas las pruebas, entre los $30 \mathrm{C}$ alumnos que tealizaron las pruebas A. B y E se encontró una cortela. ción de\} mismo orden $(.80)$. Las otras correlaciones no fue posible obtenertas at no realizat la prieba $S$.

\section{CONCLUSIONES}

Los resultados estadisticos ponen de manifiesto los siguientes puntos:

- Los resultados de la prueba de selectividad son más bajos que los obtenidos por un mismo alumno durante el curso y en el examen final.

- Existe un alto coeficiente de correiación entre la me. dia del curso (AB) y el examen final (E).

- Sin embargo entre la media del curso y el examen final, con respecto a la prueba de selectividad, el coeficiente de correlación es bajo.

- El análisis de varianza setala la no significación de medias entre los resultados de $\mathrm{AB}$ y $\mathrm{E}$, con una confianza del $99 \%$.

- Asimismo el ANOVA resalta las diferencias entre los resultados de $\mathrm{AB}$ y E, con respecto a S. Resultando significativas estas diferencias en el $95 \%$ de los casos.

Todos estos resultados nos conducen a concretar que las actuales pruebas de selectividad

- tienen una baja correlación con las actividades de. sarrolladas durante el curso,

- los conienidos del curso no están representados suficientemente en dichas pruebas, lo que implica una excesiva influencia del azar, dado el reducido número de cuestiones que se plantean,

- tienen una elevada dispersión,

- conducen a que el rendimiento del alumnado sea inferior a sus posibilidades.

Creemos que todo lo dicho hasta aqui pone de manifiesto la necesidad de un replanteamiento de la prueba de selectividad en cuanto a su extensión y contenidos, para que resulte claramente relacionada con los criterios actuales de la enseñanza de la Química.

\section{PERSPECTIVAS ABIERTAS DE TRABAJO}

Esta investigación debe continuar para responder a otra serie de aspectos mucho más importantes en relación con la propia ensentanza de la Quimica, y que podemos brevemente plantear como:

- ¿Hasta qué punto los exámenes responden en sus planteamientos al paradigma propio de la enseñanza de las Ciencias?

- iQué tipo de conocimientos, de actitudes, de capacidades y de habilidades mide realmente el exámen de selectividad?

- ¿Qué tipo de cuestiones se deben emplear?

$-i$ Cuál es el valor real selectivo de las pruebas de acceso a la Universidad?

- ¿De qué forma el tipo de prueba está condicionando el tipo de enseñanza que actualmente se da en nuestros Centros?

$\rightarrow$ ¿Qué estrategias se siguen en otras palses ante el planteamiento de los requisitos necesarios para la construcción de las pruebas de entrada a la Universidad?

ENSEÑANZA DE LAS CIENCIAS
Es

el

Qเ

te,

en

cur

gia

A!

De.

(I) $\mathrm{A}$

BLO

LAFC

ODI

$K_{i}$

(2) $\mathrm{KI}$

en

(3) $\mathrm{Al}$

(4) $A C$

$n^{\circ}$

19

19.

(6) Re

$\mathrm{Ph}$

(7) $\mathrm{DL}$

H

$\mathrm{Ph}$

ENSE: 
Estas y otras preguntas se plantean iuando abordamos el tema de la evaluación aplicada a la enschanza de la Quimica en C.O.U. Su relevancia práctica es evidenle, ya que la evaluacion, lanto en sus modelos como en sus proyecciones sobre los procesos cognitivos se en cuentra en esirecha relacion con el método o estrategia didáctica a emplear.

\section{ANEXO 1}

Descripción de cada una de las pruebas

Prueha "A»: Consta de 10 cuestiones de elección múl- liple, con 4 atternativas cada una, y 5 problemas Prueba " $B$ »: Consta de 20 cuestiones de elección múl. liple, con $\$$ alternativas cada una y 4 problemas Prueba "E»: 20 cuestiones de elección múltiple, con 4 alternativas cada una y 8 problemas.

Con las pruebas A, B y E quedaban reflejadas prácticamente todos los contenidos, pues los aspectos que no se contemplaban en la prueba de elección múltiple eran tratados en los problemas

Prueba "S": contiene dos partes, formada cada una por 1 problema y 2 cuestiones, el alumno debe elegir una de las dos partes.

\section{BIBLIOGRAFIA}

11) A cal efecto pueden consultarse:

BLOOM. 8.S. y col. 1969. eTaxonomia de los objetivos de la educaciont. El Areneo. Buenos Aires.

LAFOURCADE. P.D.: 1972, “Evaluación de los aprendizajes». Cincel. Madrid.

RODRIGUEZ DIEGUEZ, 3.L.: 1981 "Didactica General" CincelKapelusz, Madrid.

(2) KLOPFER, L.E.: "Evaluación de los aprendizajes en Ciencias", en BLOOM, B.S.: 1974, «Evaluación del aprendizaje». Troquel. Buenos Aires.

(3) AUSUBEL: 1978. "Psicotogia Educativa». Trillas.

(4) ACS-NSTA: Examination. High School Chemistry. 1977, Series $n^{\circ} 18976$ y 67426 . University of South Fiorida, USA

(5) London Examination Board (Nuffield Chemistry). 1973. JMB. 1974. Souther Univer sities Joint Board for School Examination 1982 .

(6) Resultat de la Commission Lagarigue. Butletin de f'Union des Physiciens no $\$ 97$, Octubre, 1977.

(7) DUMAS-CARRE, A.: 1980, «Reflexions du Groupe 'CHAPHAM' sur le controle des connaissance". Bulletin de l'Union de Physiciens. Octubre.
(8) Op. Cit. (7) pá 40

(9) DAVIS, I.D. y MAUSERT. 1981. "Effective Testing and Eva. luation in Chemistry" En "Source Book for Chemistry Yeachers". Six International conference on Chemical Education. August, 1981 .

(10) MATHEWS, J. y LEECE, J.R.: a A rapport on research into Nuffield Advanced Chemistry Course and Examination. Tria! Edition, 1974. pág. 35.

(11) CLEREC: 1979 y 1981: "Questionaire de Chimie. La chimie a la rentrt de l'UN1VERS1TE". Departamento de Quimica de la Universidad de Lyon.

(12) MATHEWS, J.C.: «Objetive Test in Modern Chemistry". Hutchinson, Londres, 1972.

(13) MATHEWS. J.C.: "Problems in modern Chemistry", Londres, 1971.

(14) BUTTLER, I.S.: 1976. "Problemas de Quimica" Reverte, Barcelona.

(IS) THYNE, I.M.: 1978. "Principios y iecnicas de exáment. Cot. Ciencias de la Educación. Anaya/2 pas. 123.

(16) WELLKOWITZ, J, y otros: 1981. "Estadlstica aplicada a las Ciencias de la Educacións. Santillana. Madrid. pags. 169 ss. 